\title{
Reconfiguring Experimental Archaeology using 3D Reconstruction
}

\author{
Stuart Dunn \\ Kings College London \\ Department of Digital Humanities \\ 26-29 Drury Lane \\ London, United Kingdom \\ stuart.dunn@kcl.ac.uk
}

\author{
Kirk Woolford \\ University of Sussex \\ Sussex House \\ Brighton, BN1 9RH \\ United Kingdom \\ k.woolford@sussex.ac.uk
}

\begin{abstract}
Experimental archaeology has long yielded valuable insights into the tools and techniques that featured in past peoples' relationship with the material world around them. We can determine, for example, how many trees would need to be felled to construct a large round-house of the southern British Iron Age (over one hundred), infer the exact angle needed to strike a flint core in order to knap an arrowhead in the manner of a Neolithic hunter-gatherer, or recreate the precise environmental conditions needed to store grain in underground silos over the winter months, with only the technologies and materials available to Romano-Briton villagers (see Coles 1973; Reynolds 1993). However, experimental archaeology has, hitherto, confined itself to rather rigid, empirical and quantitative questions such as those posed in these examples. This is quite understandable, and in line with good scientific practice, which stipulates that any 'experiment' must be based on replicable data, and be reproducible. Despite their potential in this area however, it is notable that digital reconstruction technologies have yet to play a significant role in experimental archaeology. Whilst many excellent examples of digital 3D reconstruction of heritage sites exist (for example the Digital Roman Forum project: http://dlib.etc.ucla.edu/projects/Forum) most, if not all, of these are characterized by a drive to establish a photorealistic re-creation of physical features. This paper will discuss possibilities that lie beyond straightforward positivist recreation of heritage sites, in the experimental reconstruction of intangible heritage.
\end{abstract}

Between 2010 and 2012, the authors led the Motion in Place Platform project (MiPP: http://www.motioninplace.org), a capital grant under the AHRC's DEDEFI scheme developing motion capture and analysis tools for exploring how people move through spaces. In the course of MiPP, a series of experiments were conducted using motion capture hardware and software at the Silchester Roman town archaeological excavation in Hampshire, and at the Butser Ancient Farm facility, where Romano-British and Iron Age dwellings have been constructed according to the best experimental practice. As well as reconstructing such Roman and early British dwellings in 3D, the authors were able to use motion capture to reconstruct the kind of activities that - according to the material evidence - are likely to have been carried out by the occupants who used them. Bespoke motion capture suits developed for the project were employed, and the traces captured and rendered with a combination of Autodesk and Unity3D software. This sheds new light on how the reconstructed spaces - and, by inference, their ancient counterparts - were most likely to have been used. In particular the exercises allowed the evaluation and visualisation of changes in behaviour which occur as a result of familiarity with an environment and the acquisition of expertise over time; and to assess how interaction between different actors affects how everyday tasks are carried out.

Motion capture, archaeology, experiment, reconstruction.

\section{INTRODUCTION}

Experimental archaeology has long yielded valuable insights into the tools and techniques that people used in the past to manipulate and navigate the material world around them. We can determine, for example, how many trees would need to be felled to construct a large round-house of the southern British Iron Age (over one hundred), infer the exact angle needed to strike a flint core in order to knap an arrowhead in the manner of a Neolithic hunter-gatherer, or recreate the precise environmental conditions needed to store grain in underground silos over the winter months, with only the technologies and materials available to Romano-British villagers (see Coles 1979; Reynolds 1993). 
However, experimental archaeology has, hitherto, confined itself to rigid, empirical and quantitative questions such as those posed in these examples. This is quite understandable, and in line with good scientific practice, which stipulates that any 'experiment' must be based on replicable data, and be reproducible. Conversely, immaterial aspects of that relationship - where people would have stood, their posture, their use of gesture, their walking speed, etc - are excluded from such experiments. This is surely a product of the context of modern archaeology, which places a premium on the object, and the material analysis and interpretation of it. This paper discusses the use of motion capture technologies in both (re)constructed and virtual historic/heritage environments, using as a case study round houses of the Romano-British iron Age. We explore these technologies as a means of reconstructing such immaterial aspects. However, rather than seeking to re-enact tasks as they would have been carried out, we seek to use motion visualisation to constrain interpretations of immaterial actions within the evidence put forward by experimental archaeology.

The question of what buildings were used for and how those uses can be identified is llongestablished, yet extremely problematic area of archaeology. It is worth noting that such questions are generally asked of high status or ritual buildings, which reflects not only the traditional preoccupations of urban archaeology, but also the fact that evidence for low-status dwellings tends not to survive as well, or be referred to in literary sources. As Percival states in his 1976 summary of evidence for Roman villas:

\begin{abstract}
Reports of villas excavated in the nineteenth, and some areas well into the twentieth, century tended to concentrate on the residential parts of the buildings, and particularly mosaics, hypocausts and architectural features such as capitals, friezes and the like. This was linked with a tendency to see the villa as a symbol of Roman luxury and comfort, so that a detailed description of each room was often accompanied by an attempt to identify its precise function and a detailed description of the kind of activities that might have taken place in it. (Percival 1976)
\end{abstract}

Our experiment sought to identify if and how valid inferences can be drawn from reconstructed and virtual environments, using the combined principles of motion data and experimental archaeology.

\section{THE MOTION IN PLACE PLATFORM PROJECT}

In 2010 and 2011, the Motion in Place Platform sought to develop tools for understanding relationships between human movement and space
(MiPP 2012). Initially, the project focused on adapting existing motion capture systems for use outside the studio. Later work on MiPP has focused on analysing and understanding motion data and its relevance to arts and humanities research by constructing (or reconstructing) and visualising narratives of immaterial human motion in material environments. Understanding motion has proven to be much more and problematic than the quantitative approaches required for digitising and/or measuring motion. This is despite the nexus implied by the common term 'material culture'. One means of beginning to understand movement is the observing and recording the movements of human beings in contemporary environments. Such methodologies have been employed in the study and conservation of heritage sites, and in museums and galleries (Guy et al 2010). There is recognition that the paths visitors use (or create themselves) to navigate around sites can be used to plan conservation practices, and to design pathways for tours and visitors to follow. However, despite numerous innovative and effective ways of gathering such data, the visualised output of such work is almost always static, in the form of maps, plotted pathways and diagrams. Ironically enough, this is the kind of static, positivist form of illustration that has been criticized by researchers who have considered the role of movement in the past and cognitive approaches to it. Witness, for example, Copeland (2009)'s critique of Ivan Margary's 1955 Roman Roads of Britain: "

Clearly the road is being treated as an abstract entity, a form of 'land art' ... which could be numbered, listed, quantified, mapped, safe and satisfying. The route of the road is extracted from the landscape, is a measured space, excluded from its surroundings both materially and cognitively' (Copeland 2009).

In general, we can observe a similar lack of sophistication in the mapping of routes far below the scale of Roman road, where such mappings exist at all: of routes between and within small settlements between and within buildings, between and points of day to day significance such as wells and hearths.

In practice, MiPP involved working together with Animazoo and researchers from the University of Sussex's Informatics lab to address one of the core issues with inertial motion capture. Inertial systems use an array of sensors placed to determine the relative angles between body parts. The Animazoo IGS-190s used for MiPP contain 19 combination gyro and magnetometers. This rotational information is mapped onto virtual skeletons to show how the user's body is positioned at a particular point in time. These systems are very 
portable. However, because of the technology they use, they do not know where the suit, or the body wearing it, is located in space. The MiPP team conducted several experiments to test additional techniques for placing the movement in space or "place". The first experiment established the utility of motion capture outside the studio, by documenting the movements of archaeologists excavating the site at Silchester, which is in a remote rural location. (figure 1 ).

Topographically, Silchester is particularly appropriate for such an experiment, as it is a single large (c. $55 \times 55$ meters) trench with a flat bottom, which reveals complex interlocking stratigraphies that become exposed as the trench floor is excavated through the Roman and Iron Age levels (Clarke et at 2007). This experiment, conducted in the 2010 field season, demonstrated that movements of contemporary people in archaeological environments can be captured, visualised and assessed with reference to the available archaeological evidence.(figure 2).

The second experiment, conducted in the spring and summer of 2011 compared movement captured in a studio with movement captured at the Butser Ancient Farm facility in Hampshire, where Romano-British and Iron Age dwellings have been constructed according to the best experimental practice (Reynolds 1993). As well as reconstructing such Roman and early British dwellings in $3 D$, the project was able to use motion capture to reconstruct the kind of activities that according to the material evidence - are likely to have been carried out by the occupants who used them. These tasks included querning (grinding flour), sweeping, fetching water (there would have been no water source inside the huts, so all water used for cooking, washing and drinking would have been

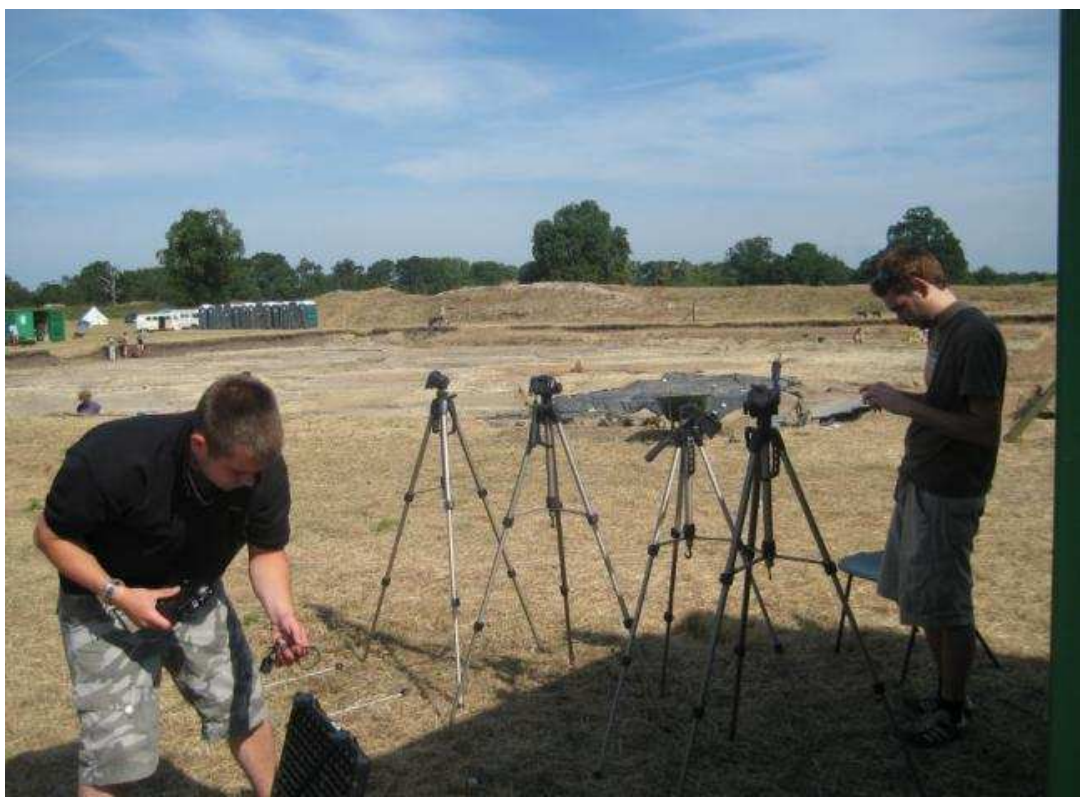

Figure 1. Experimental setup at Silchester

fetched from an external source) and bread making. The immaterial processes by which people carry out such tasks are intrinsically conditioned by their physical environment and the information they receive about it via the media of sight, smell, sound, touch and, to a lesser extent perhaps, by taste. We infer that re-recreating a round house's physical properties also involves creating the conditions which provoke cognitive responses this information.

\section{IRON AGE ROUND HOUSES}

The layout of round houses also makes them interesting environments in which to experiment with. As Webley has noted (Webley 2009), most round houses are configured with the door facing to the south east. This means that most advantageous use of day light is made, and this is generally reflected in the layout of finds from structures of this type. Finds reflecting domestic occupation, such as ceramics, loom weights and cooking paraphernalia typically cluster in the eastern section of the house, with the western section, which is often inferred to have contained sleeping quarters, relatively free of finds (Webley 2009). It has been argued that this so-called 'sunwise' model of configuration reflected not only a practical solution to the problem of round houses not containing windows, but also that it may have reflected the cycle of life and death, given that some contain burials of humans and dogs in the northeast quadrants (University of Sheffield 2012). 


\section{Reconfiguring Experimental Archaeology using 3D Reconstruction}

Stuart Dunn \& Kirk Woolford

It was not our intention to test such hypotheses in execution of domestic, and seemingly mundane, the Butser experiment, but rather to test the tasks referred

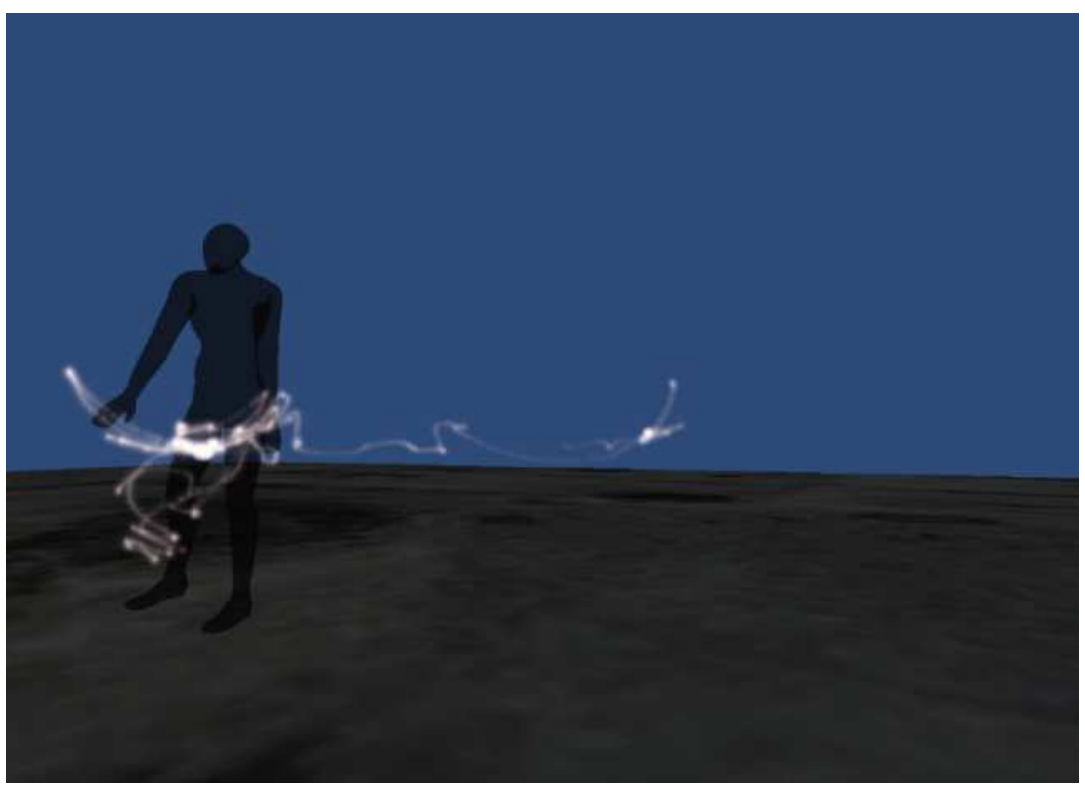

Figure 2. Motion trace rendering of an archaeologist undertaking fieldwork at Silchester

to above. We were, in essence, interested in what Eugene Ch'ng has termed 'experiential archaeology', which is explicitly differentiated from experimental archaeology by its focus on the immaterial rather than the material (Ch'ng 2009); although we would hesitate to go as far as Ch'ng and argue that advances in visual technology will make possible 'virtual time travel'.

For the purposes of the experiment, the Butser reconstruction of the round house from Moel $y$ Gerddi was used. The same bespoke motion capture suits tested at Silchester were employed, and the traces captured and rendered with a combination of Autodesk Maya, Motion Builder and Unity3D software. In addition to testing responses to information received by humans through the media of the five senses, we also sought to introduce a sixth factor, experience. In our experiment, we captured three types of person: performers who are trained to respond to with physical expressiveness to their physical environs; two students with very limited familiarity with the round house environment, and finally an experimental archaeologist who has worked at Butser for many years, and who is intimately familiar with the environment, and with the tasks involved in maintaining it.

Autodesk Motion Builder was initially used in order to plot motion trajectories of individual body parts in order to visualise the difference in movement in material versus non-material (re)recreations as well as visualising the difference between amateur or professional or informed vs. uninformed movement (see figures 3 and 4).
While the traces produced by Motion Builder are very useful for visualising movement in 3D space, Motion Builder is an animation tool, and its traces are intended to assist animators in seeing the flow of movement over time. It's not possible to measure or analyse these traces any further. The MiPP team is currently developing bespoke analysis software in order to measure such elements of movement such as the volume of space a hand passes through during an activity or the speed and frequency of movements.

This shed new light on how the reconstructed spaces - and, by inference, their ancient counterparts - were most likely to have been used. In particular the exercises allowed the evaluation and visualisation of changes in behaviour which occur as a result of familiarity with an environment. and how the acquisition of expertise over time affects the ability to navigate it; and to assess how interaction between different actors affects how everyday tasks are carried out.

\section{KNOWLEDGE AND EXPERIENCE}

That knowledge and experience of a landscape alters a human being's relationship with it has long been at the heart of so-called phenomenological archaeology (Brück 2005), and the limitations of any attempt to investigate it are well-rehearsed. One could easily argue, however, that even the most conventional analysis of high-status artefacts requires us to make value judgements about the 'quality' of the craftsmanship, and therefore the experience of the craftsman with those materials. 


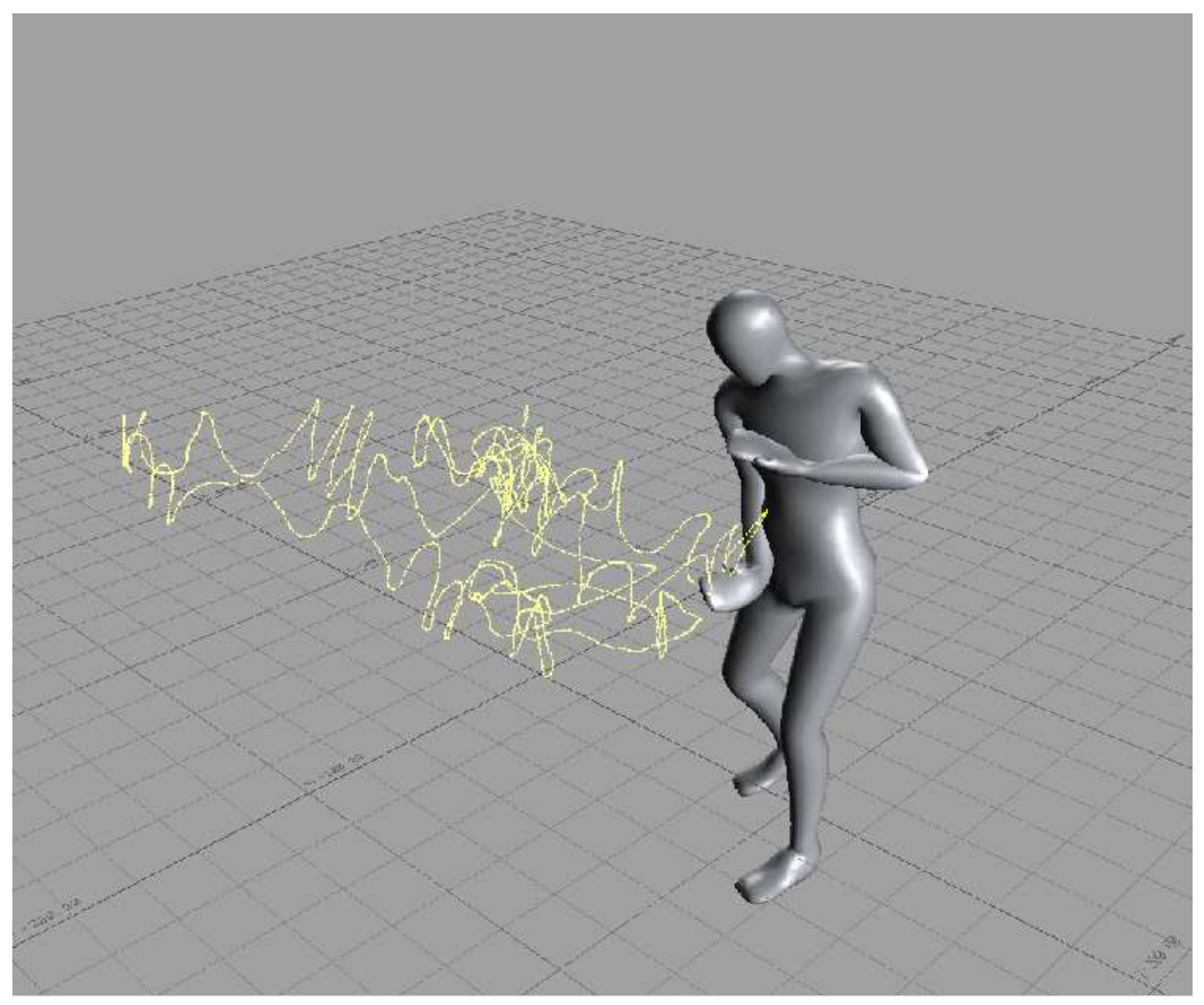

Figure 3. Motion trace of a person unfamiliar with the Moel y Gerddi round house sweeping the floor

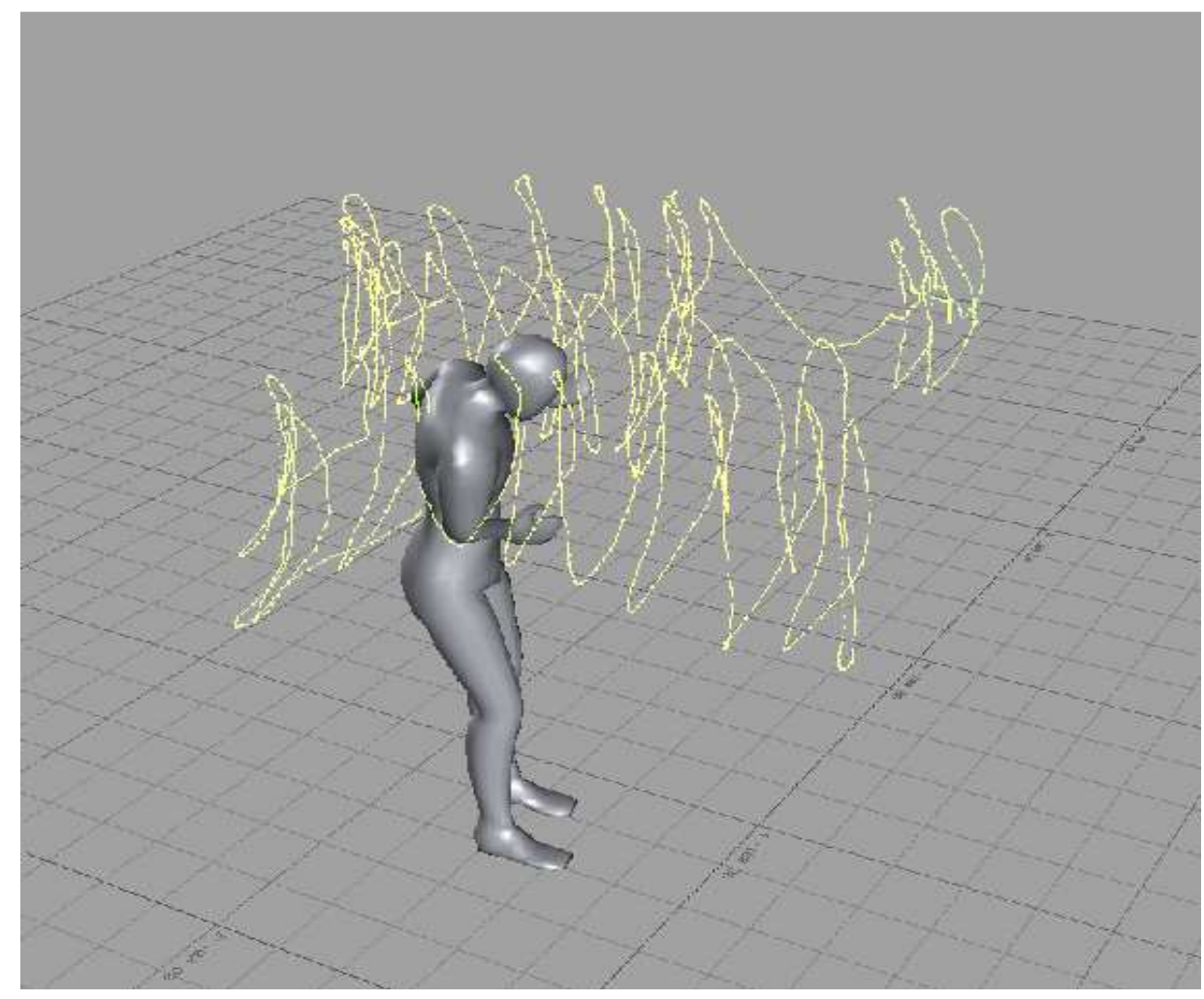

Figure 4. Motion trace of an experimental archaeologist with expert familiarity with the Moel y Gerddi round house sweeping the floor 
For the 'experience' related to manual tasks in the round house, we have no material object to examine from the past: the use of motion data allows us to create (im)material digital objects from direct observation. This allows us to make some preliminary observations about the nature of evidence that underpins $3 \mathrm{D}$ reconstruction in archaeology, and indeed the humanities more generally. We have argued elsewhere that, in general, 3D visualisations in archaeology have tended towards the positivist, with scant attention paid to the human of such spaces. We propose here that, rather than supporting the establishment of an 'experiential archaeology', the application of motion capture hardware outside the studio expands the capacity of experimental archaeology to allow documentation of the human responses to physical spaces - spaces which are, themselves, artefacts of human creation. This, we would argue, is archaeologically inferential evidence. Conceptually beneath the archaeologically inferential is the archaeologically empirical. An example of this would be the spatial footprint of the round house, which can be determined from empirical observation, and as expressed in the excavation report. We are also able to tell that the house contained twelve posts supporting its inner ring, and that it had a hearth in the centre. Empiricism and objectivity are, of course, notoriously difficult concepts to deal with in archaeology, but these are examples of statements that can be made for certain, even if one disagrees with the interpretation placed on these. A third layer is the archaeologically conjectural. Conjecture is widely used in archaeological theory and practice, and in the context of our reconstructions, we had no surfaces from which to make direct observations from which to derive textures. There is no way that we can know that the walls were the same colour, or that their surfaces had the consistency that we attributed to them. We consider that this is acceptable, so long as the lack of certainty is made explicit, and that it is divided out from our motion traces, which are inferred, and the footprint of the structure, which is empirical. This is a useful framework in which to consider 3D reconstruction in archaeology, especially where a more 'constructivist' approach is attempted, where the purpose of the reconstruction is to provoke the audience (whether that audience is public or specialist) into building its own interpretations; rather than simply presenting a positivist interpretation of the structure as a fait accompli.

In addition, the MiPP team has also explored the use of current generation "Augmented Reality" tools in order to link motion back into its environment. Qualcomm's beta augmented reality libraries (now released as "Vuforia by Qualcomm") were used to position archaeologists on overhead images of the site in Silchester in order to visualise relationships between their gestures and location, (see above, figure 2). Currently, we are working on using AR tools to place the motion captured in the Butser roundhouse into a 3D AR model to see how the space, itself, may have constrained to movement (see figure 5)

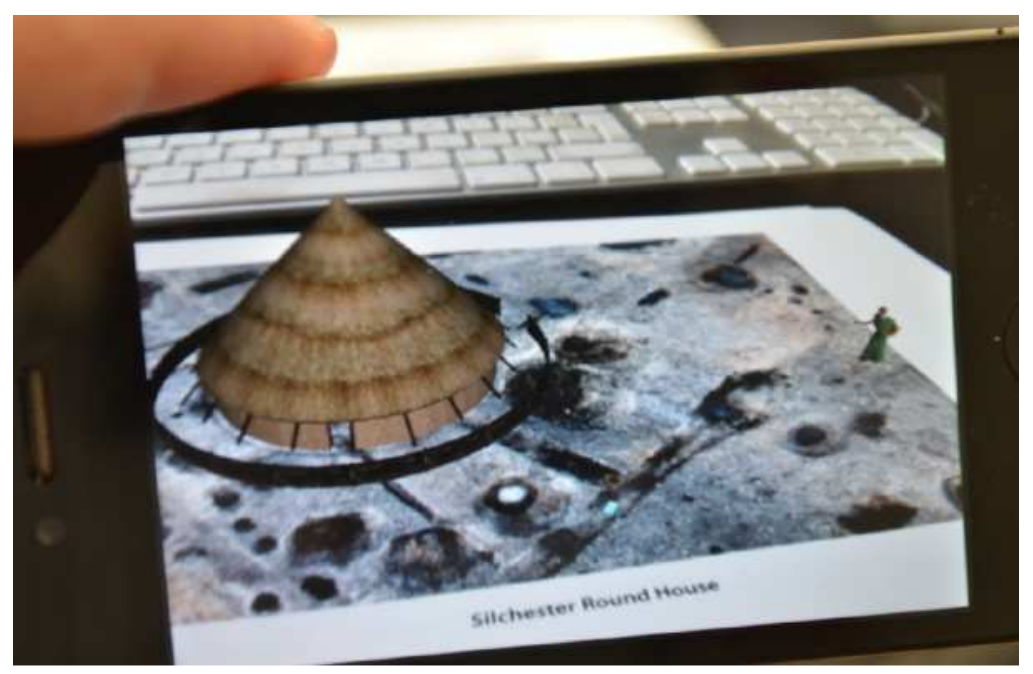

Figure 5. Augmented Reality overlay of an iron age round house

\section{MATERIALITY AND SPATIALITY}

As we have seen, experimental archaeology has a strong emphasis on the material. It shares this characteristic with other branches of archaeology, which is, after all, the study of material remains.
Materiality is an underpinning concept throughout all archaeological interpretation, and it thus influences - often unconsciously or subconsciously - those interpretations. We talk of material culture, a term which itself not unproblematic. One attribute inextricably linked with materiality is spatiality: 
every material thing exists in space and must be located somewhere. As Lewis Binford has noted:

The archaeologist "sees" the past segmentally from the perspective of fixed positions in space. The "fallout" from the events that "moved across" fixed places establishes the character of the archaeological remains on sites. To understand the past we must understand places (Binford 1982).

Our thesis in this paper is that we cannot understand places without understanding movement, and the framework of empirical, inferential and conjectural represents an approach which frees us from the 'forced' spatial certainty on (potentially) uncertain data which is implicit in many GIS approaches, and with which 3D visualisation often falls foul.

\section{CONCLUSION}

Electronic visualisation fits somewhat uneasily into the spectrum of materiality and remediation. Whilst many excellent examples of digital 3D reconstruction of heritage sites exist (for example the Digital Roman Forum project (2012) most, if not all, of these are characterized by a drive to establish a photorealistic re-creation of physical features and, as implied in the quotation from Binford above, consign movement to an implied, often even ignored, secondary status. This lacuna forms the context of the Motion in Place Platform project (MiPP: http://www.motioninplace.org), a capital grant under the AHRC's DEDEFI scheme which sought to develop tools and open debate about immaterial culture of human movement and its relationship to our understanding of material cultures.

\section{REFERENCES}

Binford, L. (1982) The archaeology of place. Journal of Anthropological Archaeology, 1(1):5-31.

Brück, J. (2005) Experiencing the past? The development of a phenomenological archaeology in
British prehistory. Archaeological Dialogues, 12(1):45-72.

Ch'ng, E. (2009) Experiential archaeology: Is virtual time travel possible? Journal of Cultural Heritage, 10(4):458-470.

Clarke, A., Fulford, M. G., Rains, M., and Tootell, K. (2007) Silchester Roman Town Insula IX: The Development of an Urban Property c. AD 40-50 c. AD 250. Internet Archaeology, 21, 2007, online at http://intarch.ac.uk/journal/issue21/silchester toc.html (retrieved 10 June 2012).

Coles, J. M. (1979) Experimental Archaeology. Academic Press Inc., London.

Copeland, T. (2009) Akeman Street: Moving through the Iron Age and Roman Landscapes. The History Press, Stroud, UK.

Digital Roman Forum project (2012) http://dlib.etc.ucla.edu/projects/Forum (retrieved 10 June 2012).

Guy, G., Dunn, S. and Gold G., (2010) Capturing Visitor Experiences for Study and Preservation. In Proceedings of Digital Humanities 2010, online at http://dh2010.cch.kcl.ac.uk/academicprogramme/abstracts/papers/html/ab-716.html (retrieved 10 June 2012).

MiPP (2012) motioninplace.org (retrieved 10 June 2012).

Reynolds, P. (1993) Experimental reconstruction. The report of a specific construction based upon the excavation of a great round house at Pimperne Down in Dorset, including an account of the life of the structure and its final dismantlement. In An Iron Age settlement in Dorset: excavation and reconstruction, Edinburgh University Monograph No. 1, 1993.

University of Sheffield (2012) http://www.sheffield.ac.uk/archaeology/research/cla dh-hallan/cladh-hallan03 (retrieved 10 June 2012).

Webley, L. 2009: Using and abandoning roundhouses. A reinterpretation of the evidence from late Bronze Age-Early Iron Age Southern England. Oxford Journal of Archaeology, 26(2):127-144. 